\title{
Diagnosis of actuator faults using LPV-gain scheduling techniques
}

\author{
Andreas Varga* Simon Hecker ${ }^{\dagger}$ \\ Daniel Ossmann ${ }^{\ddagger}$ \\ Institute of Robotics and Mechatronics, DLR Oberpfaffenhofen, D-82234 Wessling, Germany.
}

\begin{abstract}
We present a new approach for the synthesis of robust fault detection filters for the model based diagnosis of actuator faults. The underlying synthesis model is a linear parameter varying (LPV) description obtained using a combination of analytic and numerical parameter fitting techniques. The actuator LPV model contains uncertain parameters which are partly measurable, thus can be used for gain scheduling, and partly not measurable, for which robustness must be ensured. The detector synthesis approach is basically a parametric nullspace method combined with min-max parameter fitting. The synthesis technique is applied to the diagnosis of two classes of actuator faults for a civil aircraft: elevator runaway and elevator jamming at null position.
\end{abstract}

\section{Introduction}

The synthesis of fault detection filters to diagnose flight actuator faults is a challenging task in the presence of various uncertainties in the current flight conditions and aircraft parameters. Although the underlying actuator models are in general nonlinear, frequently linear parameter varying (LPV) approximations can be used to describe the actuator dynamics in a continuum of equilibrium points. These models can serve as basis for the synthesis of residual generators for fault detection, which are aimed to be robust against both measurable and unmeasurable uncertainties.

In this paper we describe firstly a methodology for the modelling of flight control actuators as LPV models depending on both known (measurable) parameters like speed, altitude and surface deflection, but also on unknown (uncertain) aircraft parameters like mass or position of center of gravity. A quasi-LPV representation for a detailed nonlinear actuator model, which is partly based on black-box models is derived. For several grid-points in the flight envelope and the weight and balance domain of the aircraft, the coefficients of the quasi-LPV model are identified using time histories of the detailed nonlinear simulation model. Finally, a multivariable polynomial fitting is applied to the grid-point models to obtain a globally valid quasi-LPV model of the actuator that is of high accuracy and based on a simple structure such that it can be easily implemented in the flight control computer.

In the second part, we use the developed LPV models to synthesize residual generation filters to serve for actuator fault detection purposes. These filters are designed to be robust against both measurable and unmeasurable parameters using recently developed robust synthesis methods relying on a combination of symbolic and numerical methods. Two challenging fault detection examples are considered to illustrate the proposed approach for a civil aircraft: the detection of elevator runaway and jamming at null position.

\footnotetext{
*Senior Scientist, Department of System Dynamics and Control, Institute of Robotics and Mechatronics, DLR Oberpfaffenhofen, D-82234 Wessling, Germany; Andreas.Varga@dlr.de

${ }^{\dagger}$ Professor, Department of Electrical Engineering and Information Technology, University of Applied Sciences Munich, D80335 Munich, Germany; Simon.Hecker@hm.edu

${ }^{\ddagger}$ Research Associate, Department of System Dynamics and Control, Institute of Robotics and Mechatronics, DLR Oberpfaffenhofen, D-82234 Wessling, Germany; Daniel.Ossmann@dlr.de
} 


\section{LPV model generation for the nonlinear actuator model}

In this section, we describe the development of an LPV model for a system formed from an actuator and the associated elevator control surface which is part of the ADDSAFE benchmark model ${ }^{\mathrm{a}}$. The resulting elevator actuator model has a first order LPV-system representation of the form

$$
\begin{aligned}
& \dot{x}=-k(\rho) x+k(\rho) u, \\
& y=x,
\end{aligned}
$$

where $x$ and $y$ are the rod position and $u$ is the commanded position. The gain $k(\rho)$ generally depends on both measurable and unmeasurable parameters contained in a vector $\rho$. Typical values of the gain are around $k=14$.

The aim of LPV model generation is to determine, starting from a high fidelity nonlinear actuator model, the corresponding LPV-approximation of the form (1), with an explicit parametric expression of $k(\rho)$. This model will then serve for synthesis purposes of robust fault detection filters to detect several categories of actuator faults.

\section{A. Detailed actuator model}

The detailed actuator model represents a simplified, nonlinear first order equation of an hydraulic servo controlled actuator ${ }^{1}$ which is widely used in today's aircraft. The equations are of the form

$$
\dot{x}=K_{c i} K_{p}(u-x) \sqrt{\frac{\Delta P(x)-\frac{F_{a e r o}(p, x, \dot{x})}{S}}{\Delta P_{\text {ref }}+\frac{K_{a}(x) \dot{x}^{2}}{S}}},
$$

where $K_{p}$ is the servo control gain, $K_{c i}$ is a gain to convert an estimated current to a corresponding rod speed, $\Delta P$ is the hydraulic pressure delivered to the actuator, $\Delta P_{r e f}$ is a differential pressure for a fully opened servo-valve (maximum rod speed), $F_{\text {aero }}$ represents the aerodynamic forces at the control surface, $K_{a}(x) \dot{x}^{2}$ represents the estimated servo-control load of the adjacent actuator in damping mode and $S$ is the actuator piston surface area. $p=\left(V_{c a s}, h, m, X_{c g}\right)$ is a vector of aircraft and flight condition parameters including the calibrated airspeed $V_{\text {cas }}$, the aircraft altitude $h$, the aircraft mass $m$ and the position of the center of gravity $X_{c g}$ along the $x$-axis.

\section{B. Quasi-LPV approximation of the detailed actuator model}

In order to get a simple quasi-LPV model approximating (2) with good accuracy, the idea is to cover the parameter varying dynamic behavior in a simple function $K(p, x, \dot{x})$, which is then used in a first order actuator model as given in (1).

\section{Physical considerations for derivation of model structure}

From various investigations it was obvious that the variations in the dynamic behavior (variation of $K$ ) mainly come from the aerodynamic force $F_{\text {aero }}$ that acts on the control surface, where $F_{\text {aero }}$ itself usually depends on the aircraft parameters $p$, the actuator position $x$ and the sign of the actuator position rate $\dot{x}$. The influence of $x$ and $\operatorname{sign}(\dot{x})$ can be explained by investigating the actuator dynamics for different control surface positions and signs of deflection rate (see Figure 1). Assuming, that for a deflection angle of zero of the control surface (middle part of Figure 1 ) the force $F_{\text {aero }}$ is also zero, then for small deflections the dynamics of the actuator may be accurately described by the constant gain $K=K_{1}$, independent of deflecting the control surface up- or downwards. In case of an upward deflected control surface (upper part of Figure 1) we assume that $F_{\text {aero }}$ is not zero and typically this force will try to push the control surface back to a deflection angle of zero. Hence, if a small additional upward deflection is commanded, the actuator has to work against the disturbing force $F_{\text {aero }}$ and therefore the dynamics will be slower compared to a small downward deflection where $F_{\text {aero }}$ supports the actuator in deflecting the control surface towards zero. Therefore for each situation (zero or upward initial deflection combined with additional up- or downward

\footnotetext{
${ }^{a}$ ADDSAFE is the acronym of the European research project Advanced Fault Diagnosis for Safer Flight Guidance and Control - AAT-2008-RTD-233815 (2009-2012).
} 

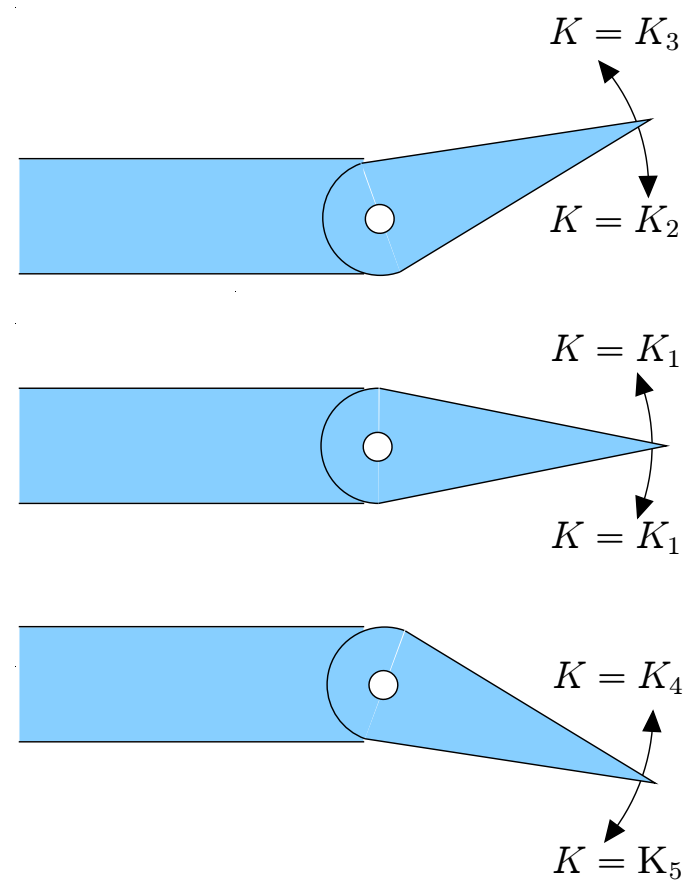

Figure 1. Actuator gains for different deflections angles and signs of deflection rate

perturbation) one may derive different LTI models by linearization of (2). For the detailed actuator model under investigation we obtained the result that $K_{3}<K_{1}<K_{2}$, which exactly follows the interpretation above. In addition, if $F_{\text {aero }}$ is symmetric to a deflection angle of zero we may obtain that $K_{5} \approx K_{3}$ and $K_{4} \approx K_{2}$.

Following the above discussion and assuming that the aircraft parameters $p$ are fixed, a suitable description for $K$ was derived as

$$
K(x, \operatorname{sign}(\dot{x}))=C_{0}+C_{1} \operatorname{sign}(\dot{x})\left(x+C_{2}\right),
$$

where $C_{0}$ may be interpreted as the nominal gain, $C_{1}$ describes the influence of the deflection angle $x$ on $K$ and $\operatorname{sign}(\dot{x})$ allows to distinguish between upward and downward movements of the control surface. $C_{2}$ may be interpreted as a position offset, as the assumption that $F_{\text {aero }}$ is zero at zero deflection angle is not true in reality.

\section{Derivation of model coefficients using optimization}

The coefficients $C_{0}, C_{1}$ and $C_{2}$ are obtained by solving

$$
\min _{C_{0}, C_{1}, C_{2}}\left(\frac{1}{n-1} \sum_{i=1}^{n}\left(x_{r}\left(t_{i}\right)-x_{L P V}\left(t_{i}\right)\right)^{2}\right)^{\frac{1}{2}},
$$

where $x_{r}\left(t_{i}\right)$ denotes the output of the detailed nonlinear actuator model (2) at time $t_{i}, x_{L P V}\left(t_{i}\right)$ is the output of the quasi-LPV model (1) at time $t_{i}$ with $K$ given as (3) and $n$ is the number of equidistant time steps of a simulation with various input commands $u(t)$.

\section{Derivation of globally valid model using affine polynomial fitting}

As $F_{\text {aero }}$ strongly depends on the actual position in the flight envelope and the aircraft condition, we will obtain different values for $C_{0}, C_{1}$ and $C_{2}$ for different parameters $p$. To include also the dependency on $p$ in the quasi-LPV model, we generated optimal values for $C_{0}, C_{1}$ and $C_{2}$ by solving (4) for a grid of parameter values $p$ as given in Table 1 . In order to get a globally valid model, an affine polynomial fitting of the optimal 


\begin{tabular}{ccccc}
\hline Grid Point & $m[t]$ & $X_{c g}[\%]$ & $h[f t]$ & $V_{\text {cas }}[k t s]$ \\
\hline 1 & 120 & 21 & 0 & 154.6 \\
2 & 120 & 21 & 0 & 275 \\
3 & 120 & 21 & 37000 & 176.1 \\
4 & 120 & 21 & 37000 & 275 \\
5 & 120 & 38 & 0 & 154.6 \\
6 & 120 & 38 & 0 & 275 \\
7 & 120 & 38 & 37000 & 176.1 \\
8 & 120 & 38 & 37000 & 275 \\
9 & 200 & 21 & 0 & 190.5 \\
10 & 200 & 21 & 0 & 275 \\
11 & 200 & 21 & 37000 & 229.6 \\
12 & 200 & 21 & 37000 & 275 \\
13 & 200 & 38 & 0 & 190.5 \\
14 & 200 & 38 & 0 & 275 \\
15 & 200 & 38 & 37000 & 229.6 \\
16 & 200 & 38 & 37000 & 275 \\
\hline
\end{tabular}

Table 1. Grid point values of parameters

grid-point coefficients was applied using the methods from, ${ }^{3}$ resulting in a polynomial description of the form

$$
C_{i}=C_{i}(p)=C_{i, 0}+C_{i, m} m+C_{i, X_{c} g} X_{c g}+C_{i, h} h+C_{i, V_{c a s}} V_{c a s}, i=0, \ldots, 2 .
$$

A simulative validation of the model at the grid point flight parameters has shown that the simple affine polynomial approximation of the optimal grid point values allowed to have a good model accuracy. Hence, no higher order approximations were generated. In all cases we could even neglect some of the coefficients in (5) as they had almost no influence. An investigation of the derived polynomial coefficients revealed that $V_{\text {cas }}$ has a strong influence on the actuator dynamics as $F_{\text {aero }}$ significantly depends on this variable.

Finally, a quasi-LPV model of the form $(1)$, with $K=k(\rho)$ and $\rho=(x, \operatorname{sign}(\dot{x}), p)$ was derived, which is suitable for fault diagnosis applications and simple enough to be implemented in the aircraft flight control computer.

\section{Simulation results}

In Figures 2-3 some simulation results are shown, where $x_{r}$ is the actuator position of the detailed nonlinear model, $x_{L P V}$ the actuator position of the quasi-LPV model, $x_{c}$ the actuator position of a simple actuator model with fixed $K=1 / 0.07$. $e_{L P V}=x_{r}-x_{L P V}$ and $e_{c}=x_{r}-x_{c}$ are the corresponding position errors. Simulations with small and large control surface deflections are shown and in both cases a remarkable reduction of the position error can be seen when using the quasi-LPV model instead of the simple model with constant gain $K$. 

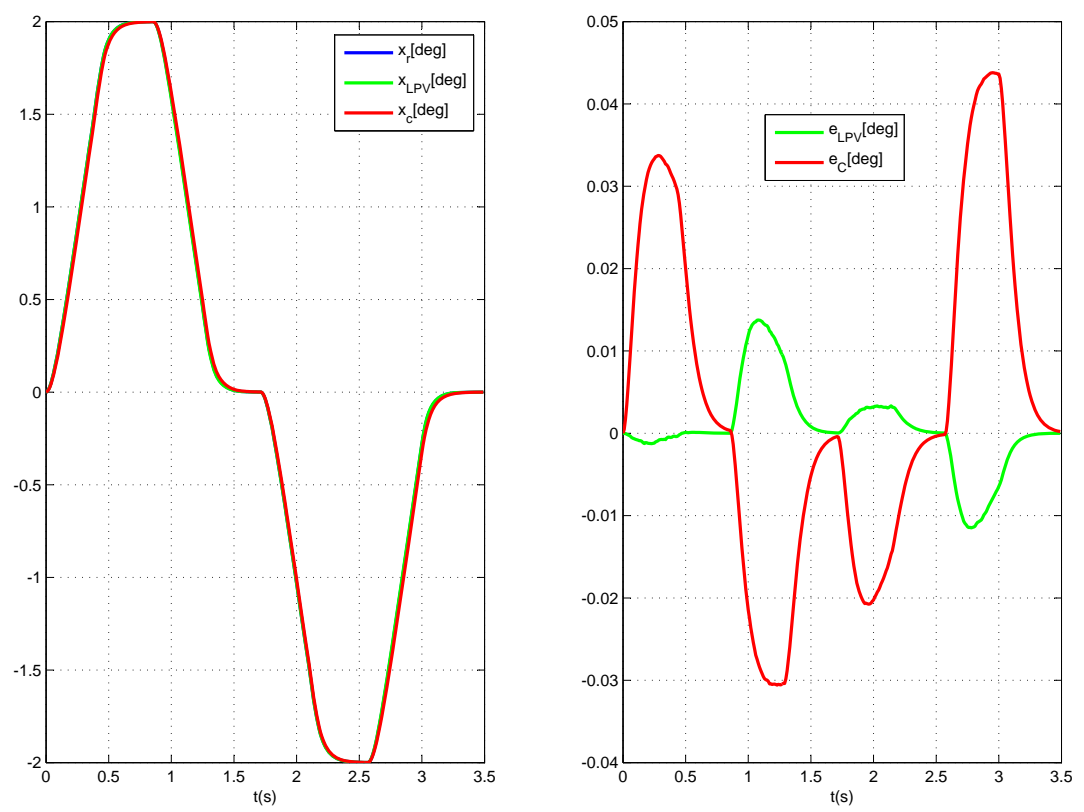

Figure 2. Simulation: elevator, grid point 9, small deflection
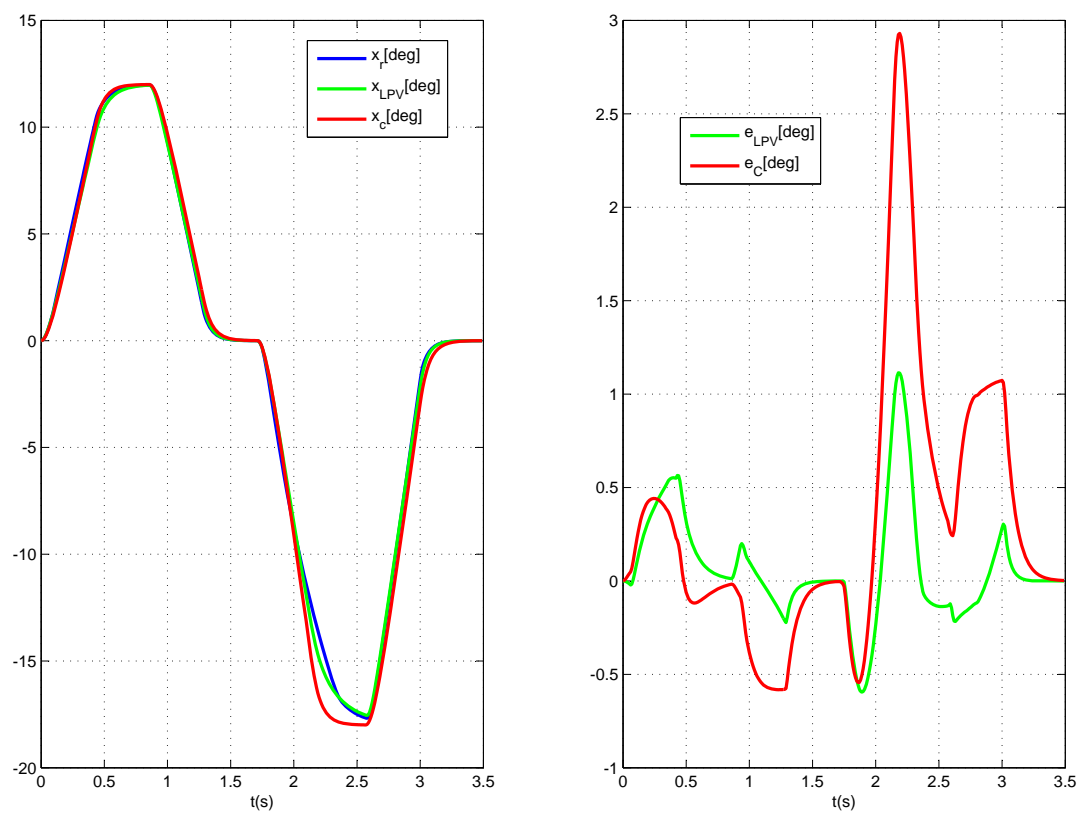

Figure 3. Simulation: elevator, grid point 3, large deflection 


\section{LPV Detector Synthesis}

\section{A. The robust actuator fault detection problem}

In what follows we will assume that the parameters are constant. Thus, we can alternatively use an inputoutput representation of the actuator fault model in the form

$$
\mathbf{y}(s)=G_{u}(s, \rho) \mathbf{u}(s)+G_{u}(s, \rho) \mathbf{f}(s),
$$

where $\mathbf{y}(s), \mathbf{u}(s)$, and $\mathbf{f}(s)$ are the Laplace-transformed quantities of $y(t), u(t)$, and $f(t)$, respectively, and $G_{u}(s, \rho)$ is the parameter dependent transfer function from the control input to output as well as from the fault input to output. $G_{u}(s, \rho)$ corresponding to (1) is given by

$$
G_{u}(s, \rho)=\frac{k(\rho)}{s+k(\rho)}
$$

Regarding the unknown parameter vector $\rho$, generally we can assume that it has two components: $\rho_{1} \in$ $\Pi_{1}$, which is not measurable, and $\rho_{2} \in \Pi_{2}$, which is measurable, and thus $\rho \in \Pi:=\Pi_{1} \times \Pi_{2}$. In the case of the elevator actuator model $\rho_{1}=\left(m, X_{c g}\right)$, while $\rho_{2}=\left(x, \operatorname{sign}(\dot{x}), V_{\text {cas }}, h\right)$. The synthesis problem formulated bellow attempts to solve basically a robust fault detection problem with respect to $\rho_{1}$, while taking advantage of the availability of $\rho_{2}$ by attempting to achieve robustness using an LPV gain scheduling approach.

A linear residual generator (or fault detection filter) processes the measurable system outputs $y(t)$ and control inputs $u(t)$ and generates the residual signals $r(t)$ which serve for decision making on the presence or absence of faults. We use a parameter dependent filter of the form

$$
\mathbf{r}(s)=Q\left(s, \rho_{2}\right)\left[\begin{array}{l}
\mathbf{y}(s) \\
\mathbf{u}(s)
\end{array}\right],
$$

where $Q\left(s, \rho_{2}\right)$ is the $1 \times 2$ transfer-function matrix of the filter, which explicitly depends on the measurable parameter $\rho_{2}$ (e.g., via an equivalent state-space realization of the filter). For a physically realizable filter, $Q\left(s, \rho_{2}\right)$ must be proper with respect to $s$ (i.e., only with finite poles) and robustly stable (i.e., only with poles having negative real parts for all values of $\rho_{2}$ ).

The residual signal $r(t)$ in (8) generally depends via the system outputs $y(t)$ on all system inputs $u(t)$ and $f(t)$. The residual generation system, obtained by replacing in (8) $\mathbf{y}(s)$ by its expression in (6), is given by

$$
\mathbf{r}(s)=R_{u}(s, \rho) \mathbf{u}(s)+R_{f}(s, \rho) \mathbf{f}(s)
$$

where

$$
\left[R_{u}(s, \rho) \mid R_{f}(s, \rho)\right]:=Q\left(s, \rho_{2}\right) G_{e}(s, \rho)
$$

with

$$
G_{e}(s, \rho):=\left[\begin{array}{c|c}
G_{u}(s, \rho) & G_{u}(s, \rho) \\
1 & 0
\end{array}\right]
$$

For a successfully designed filter $Q\left(s, \rho_{2}\right)$, the corresponding residual generation system is proper with respect to variable $s$, robustly stable and achieves specific fault detection requirements (e.g., exact or approximate decoupling of control inputs from the residuals).

We can now formulate the following Robust Fault Detection Problem (RFDP): For the LPV-system (6), determine a proper and stable linear residual generator (or fault detector) filter having the form (8) such that for all $\rho \in \Pi$ and a given $\gamma>0$ there exists $\beta>0$ such that

(i) $\quad\|r(t)\| \leq \gamma\|u(t)\|$ when $f(t)=0$ and for all $u(t)$;

(ii) $\quad\|r(t)\| \geq \beta\|f\|$ for $u(t)=0$

(iii) $\quad r(t)$ is asymptotically bounded.

Here $\|\cdot\|$ denotes an appropriate signal norm. The gap defined as $\beta / \gamma$ measures the sensitivity of detection task, where larger values guarantees the detection of smaller faults. The exact solution of the RFDP corresponds to the case when we can choose $\gamma=0$, and the corresponding gap is $\infty$. 
The fulfillment of requirement $(i i)$ ensures that faults produce non-zero residual responses. When designing fault detectors, this requirement for fault detectability is usually replaced by the stronger condition that persistent (e.g., constant) faults produce asymptotically persistent residuals. This requirement is known as strong fault detectability.

The requirements $(i)$ and $(i i)$ can be easily transcribed into equivalent synthesis conditions. The approximate decoupling condition $(i)$ requires that $R_{u}(s, \rho)$ has a small norm $\forall \rho$, and thus can be achieved by minimizing the worst-case norm

$$
\max _{\rho \in \Pi}\left\|R_{u}\left(s, \rho_{2}\right)\right\|
$$

With a suitable scaling of the detector, we can always achieve that this worst-case norm is equal to a given $\gamma$. The (detectability) condition (ii) is equivalent to

$$
\beta:=\min _{\rho \in \Pi}\left\|R_{f}(s, \rho)\right\|>0 .
$$

Additionally, the condition (iii) on the boundedness of the residual signal requires that $R_{f}(s, \rho)$ as well as $R_{u}(s, \rho)$ are stable transfer functions for all $\rho$.

In the next subsection a synthesis procedure to solve the formulated RFDP is addressed using a combination of symbolic and numerical computational tools. In this paper, we specialize this procedure by providing explicit analytical solutions of the RFDP for two categories of actuator faults.

\section{B. Parametric nullspace method}

Assume temporarily that $\rho$ is measurable and let $Q(s, \rho)$ be a detector which exactly solves the RFDP. Thus, $Q(s, \rho)$ is stable and proper and for all $\rho \in \Pi$ satisfies

$$
Q(s, \rho) G(s, \rho)=0
$$

where

$$
G(s, \rho)=\left[\begin{array}{c}
G_{u}(s, \rho) \\
1
\end{array}\right]
$$

and

$$
R_{f}(s, \rho) \neq 0 .
$$

From (14) it appears that $Q(s, \rho)$ is a left annihilator of $G(s, \rho)$. The synthesis method proposed by Varga $^{5}$ determines $Q(s, \rho)$ symbolically to serve for obtaining an approximation $\bar{Q}\left(s, \rho_{2}\right)$ by minimizing, for example, the worst-case error norm

$$
\max _{\rho \in \Pi}\left\|Q(s, \rho)-\bar{Q}\left(s, \rho_{2}\right)\right\|
$$

such that conditions (13) for $Q(s, \rho)=\bar{Q}\left(s, \rho_{2}\right)$ jointly with the stability requirement on $R_{u}(s, \rho)$ and $R_{f}(s, \rho)$ are fulfilled. Possible norms to be employed in (17) are the $\mathcal{H}_{2}$ - or $\mathcal{H}_{\infty}$-norms, ${ }^{6}$ or even the $\nu$-gap norm. ${ }^{7}$ Alternatively, the weighted worst-case error norm

$$
\max _{\rho \in \Pi}\left\|\left(Q(s, \rho)-\bar{Q}\left(s, \rho_{2}\right)\right) G_{e}(s, \rho)\right\|
$$

can be minimized to fulfill both conditions (12) and (13).

The result of the nullspace computation based approach is a parametric state-space realization for $Q(s, \rho)$ of the form

$$
Q(s, \rho)=\left[\begin{array}{c|c}
A_{Q} & B_{Q}(\rho) \\
\hline C_{Q} & D_{Q}(\rho)
\end{array}\right],
$$

where $A_{Q}$ and $C_{Q}$ are constant matrices, and only $B_{Q}(\rho)$ and $D_{Q}(\rho)$ depend on $\rho$.

The realization of the transfer function from the fault to the residual $R_{f}(s, \rho)$ can be also explicitly obtained in the form

$$
R_{f}(s, \rho)=\left[\begin{array}{c|c}
A_{Q} & \widetilde{B}_{f}(\rho) \\
\hline C_{Q} & \widetilde{D}_{f}(\rho)
\end{array}\right],
$$

thus sharing the constant matrices $A_{Q}$ and $C_{Q}$. 


\section{Computation of approximative detectors}

In the case when $\rho$ contains both non-measurable components $\rho_{1}$ as well as measurable ones $\rho_{2}$ we can use the computed $Q(s, \rho):=Q\left(s, \rho_{1}, \rho_{2}\right)$ to obtain a suitable approximation $\bar{Q}\left(s, \rho_{2}\right)$ depending only of $\rho_{2}$. The solution of the approximation problems (17) or (18) can be addressed by exploiting the form of the realization (19) by choosing $\bar{Q}\left(s, \rho_{2}\right)$ to share the constant matrices $A_{Q}$ and $C_{Q}$

$$
\bar{Q}\left(s, \rho_{2}\right)=\left[\begin{array}{l|l}
A_{Q} & \bar{B}_{Q}\left(\rho_{2}\right) \\
\hline C_{Q} & \bar{D}_{Q}\left(\rho_{2}\right)
\end{array}\right]
$$

There are several possibilities to robustly fit $\bar{B}_{Q}\left(\rho_{2}\right)$ and $\bar{D}_{Q}\left(\rho_{2}\right)$ to $B_{Q}\left(\rho_{1}, \rho_{2}\right)$ and $D_{Q}\left(\rho_{1}, \rho_{2}\right)$, respectively. We mention a few of them in what follows:

Structure preserving fitting: This involves finding a value $\bar{\rho}_{1}$ of $\rho_{1}$ which produces the best approximation $\bar{B}_{Q}\left(\rho_{2}\right):=B_{Q}\left(\bar{\rho}_{1}, \rho_{2}\right)$, by solving

$$
\bar{\rho}_{1}=\arg \min _{z \in \Pi_{1}} \max _{\rho \in \Pi}\left\|B_{Q}\left(\rho_{1}, \rho_{2}\right)-B_{Q}\left(z, \rho_{2}\right)\right\|
$$

Similar computation is performed for $\bar{D}_{Q}\left(\rho_{2}\right)$.

Parameter fitting: We can assume for $\bar{B}_{Q}\left(\rho_{2}\right)$ a certain parametric form $\widetilde{B}_{Q}\left(\rho_{2}, \theta\right)$ (e.g., affine, polynomial, rational, etc.) and fit the free parameters $\theta$ by solving

$$
\bar{\theta}=\arg \min _{\theta} \max _{\rho \in \Pi}\left\|B_{Q}(\rho)-\widetilde{B}_{Q}\left(\rho_{2}, \theta\right)\right\|
$$

and define $\bar{B}_{Q}\left(\rho_{2}\right):=\widetilde{B}_{Q}\left(\rho_{2}, \bar{\theta}\right)$ (and similarly for $\left.\bar{D}_{Q}\left(\rho_{2}\right)\right)$.

Input-output fitting: The fitting is performed by globally minimizing a suitable worst-case system norm

$$
\max _{\rho \in \Pi}\left\|Q(s, \rho)-\bar{Q}\left(s, \rho_{2}\right)\right\|
$$

For $\bar{B}_{Q}\left(\rho_{2}\right)$ and $\bar{D}_{Q}\left(\rho_{2}\right)$ in (20) either a structure preserving form or a parametric form can be used.

Weighted input-output fitting: The fitting is performed by globally minimizing the worst-case weighted system norm

$$
\max _{\rho \in \Pi}\left\|\left(Q(s, \rho)-\bar{Q}\left(s, \rho_{2}\right)\right) G_{e}(s, \rho)\right\|
$$

For $\bar{B}_{Q}\left(\rho_{2}\right)$ and $\bar{D}_{Q}\left(\rho_{2}\right)$ in (20) either a structure preserving form or a parametric form can be used.

When using standard optimization tools to solve the above min-max parameter fitting problems, the evaluation of the above criteria involves performing a worst-case optimization-based search. Thus, function evaluations are potentially expensive, and therefore an alternative is to replace the semi-infinite optimization problems by computationally tractable finite dimensional optimization problems. A frequently used approximation method is to use instead the continuous domain $\Pi$ only a discrete set of points $\Pi_{N}=$ $\left\{\rho^{(1)}, \rho^{(2)}, \ldots, \rho^{(N)}\right\}$, obtained, for example, by parameter gridding. By using a sufficiently dense grid of points, it is expected to obtain a satisfactory approximation of the continuous-case worst-case. One advantage of the gridding based approach is the possibility to perform in parallel all function evaluations necessary for the determination of worst-case maximum.

\section{Fault detection and diagnosis system for fault identification}

In this section we discuss the setup of a typical fault detection and diagnosis (FDD) system, used to detect and identify different types of actuator faults. Such a FDD system is depicted in Fig. 4, where besides the residual generator, blocks for residual evaluation, decision making and fault identification are present. Fault identification involves the determination of the type of the fault signal $f$ from the generated residual signal $r$ and the measured output $y$ and controlled input $u$ signals. Typical actuator faults to be identified 


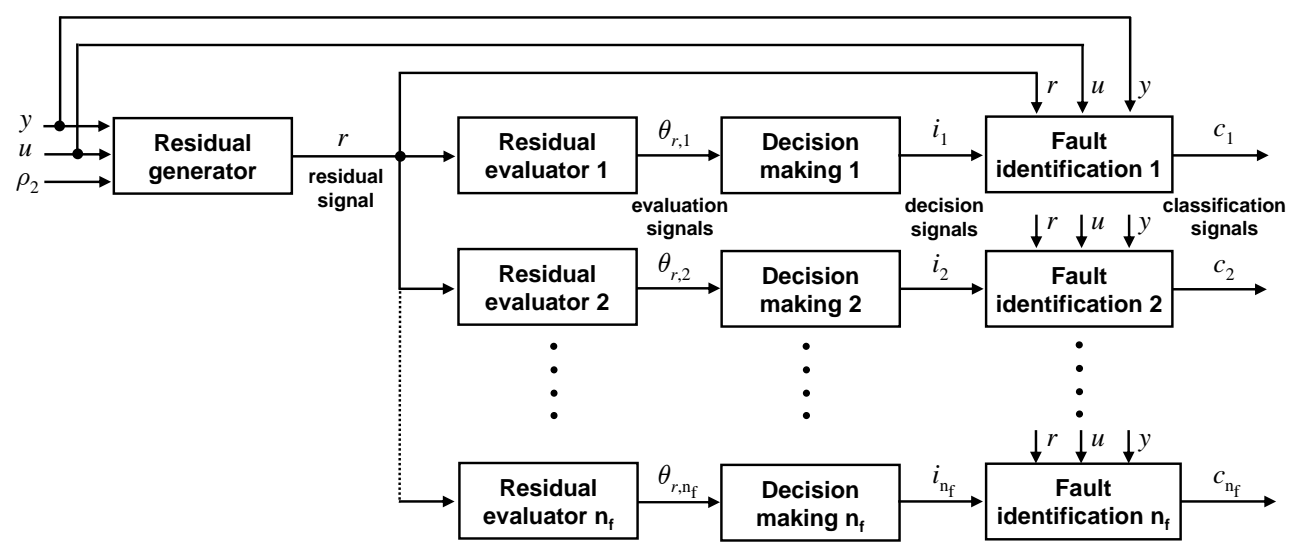

Figure 4. FDD system structure for actuator fault identification

are jamming, runaway, oscillatory failure, etc. The determination of the fault characteristics is crucial for triggering an adequate reconfiguration of the actuation systems (e.g., switching to a backup actuator) or even of the flight control laws (e.g., by changing the control distribution strategy).

The FDD structure in Fig. 4 includes only a single residual generator for fault detection (or simply, a fault detector), which processes the commanded actuator position $u$ and the measured current actuator position $y$ and generates the scalar residual signal $r$. For the robust fault detection, a single LPV detector is sufficient, where $\rho_{2}$ is the scheduling variable as described in section III. For the identification of different types of faults, individual post processing of the residual is performed, by taking into account the different possible shapes of fault signals as well as the specific requirements on detection times. For $n_{f}$ different types of fault signals to be detected, a multi-channel structure is used for the residual evaluation, decision making and fault identification. For each fault type to be identified, a residual evaluation block and decision making block is present, whose parameters are chosen to reflect the characteristics of the assumed fault signal. Thus, for the $j$-th type of fault, an approximation $\theta_{r, j}$ of the residual norm $\|r\|$ is computed in the evaluation block. Then, this value is used in the threshold-based decision making block to generate the decision signal $i_{j}$, which, if nonzero, triggers a signal based fault identification process. Each identification block provides qualitative and quantitative information of the faults by processing the input and output signals $u$ and $y$ of the actuator, as well as the residual signal $r$. The output of this block is the fault classification signal $c_{j}$, which can be used to trigger specific control reconfiguration actions.

Within this paper we use a structure with only two channels, to generate the fault classification signals for actuator jamming in null position $\left(c_{1}\right)$ and actuator runaway $\left(c_{2}\right)$. However, the structure can easily be extended with additional channels for the detection of other actuator faults, as for example, actuator jamming in nonzero position or oscillatory faults.

\section{A. Residual generation filter synthesis}

The synthesis method presented section III has been applied to address two fault scenarios of the ADDSAFE Benchmark: the elevator runaway and elevator jamming at null position. Assuming all components of $\rho$ entering in the model (7) are measurable (i.e., $\rho_{2}=\rho$ ), both problems can be addressed using a first order detector of the form

$$
Q(s, \rho)=\left[\begin{array}{ll}
\frac{a}{k_{0}} \frac{s+k(\rho)}{s+a} & -\frac{k(\rho) a}{k_{0}(s+a)}
\end{array}\right],
$$

where $a$ is an arbitrary positive value specifying the dynamics of the detector and $k_{0}$ is a nominal value of the gain $k(\rho)$. This detector leads to an exact decoupling of control inputs in (10), thus $R_{u}(s, \rho)=0$, and to the fault-to-residual transfer function

$$
R_{f}(s, \rho)=\frac{k(\rho)}{k_{0}} \frac{a}{s+a}
$$


Thus, the residual signal provides a filtered estimation of the fault multiplied with $\frac{k(\rho)}{k_{0}}$, with the property that $\lim _{t \rightarrow \infty} r(t)=\frac{k(\rho)}{k_{0}} \lim _{t \rightarrow \infty} f(t)$. This allows to easily reconstruct the actuator fault signal $f$ for further use in fault identification.

The corresponding state-space realization of the residual generator has the matrices

$$
A_{Q}=-a, \quad B_{Q}(\rho)=a\left[\frac{k(\rho)-a}{k_{0}} \frac{k(\rho)}{k_{0}}\right], \quad C_{Q}=1, \quad D_{Q}=\left[\begin{array}{cc}
\frac{a}{k_{0}} & 0
\end{array}\right]
$$

The chosen form (22) of the detection filter leads to a state-space realization with a constant feed-through matrix $D_{Q}$. This has the major advantage to prevent all direct effects on $r$ of the discontinuities in the scheduling signal $\rho_{2}$ (e.g., jumps due to the presence of the signum-function in (3)).

In the case when the uncertain components in $\rho_{1}$ are present, we use a similar detector as in (22), but with $k(\rho)$ replaced by an approximation $\widetilde{k}\left(\rho_{2}\right)$, determined such that the error $\delta=k\left(\rho_{1}, \rho_{2}\right)-\widetilde{k}\left(\rho_{2}\right)$ is minimized over all $\rho_{1}$ and $\rho_{2}$ values. When employing $\widetilde{k}\left(\rho_{2}\right)$, the resulting transfer functions from $u$ to $r$ and $f$ to $r$ can be expressed as

$$
R_{u}(s, \rho)=\frac{a s \delta}{k_{0}(s+a)\left(s+\widetilde{k}\left(\rho_{2}\right)+\delta\right)}, \quad R_{f}(s, \rho)=\frac{a\left(\widetilde{k}\left(\rho_{2}\right)+\delta\right)\left(s+\widetilde{k}\left(\rho_{2}\right)\right)}{k_{0}(s+a)\left(s+\widetilde{k}\left(\rho_{2}\right)+\delta\right)}
$$

It follows, that as long as $\delta$ is sufficiently small, a satisfactory decoupling of inputs can be achieved simultaneously with the detection of faults.

\section{B. Residual evaluation and decision making}

The evaluation of the residual signal often requires the computation of a measure of the residual signal energy, for which the 2-norm of the signal is usually an appropriate choice. The so called Narendra signal evaluation scheme can be used in the form

$$
\theta_{r, j}(t)=\alpha_{r, j}\|r(t)\|_{2}+\beta_{r, j} \int_{0}^{t} e^{-\gamma_{r, j}(t-\tau)}\|r(\tau)\|_{2} d \tau,
$$

where $\theta_{r, j}(t)$ can be generated by the first order differential equation

$$
\begin{aligned}
& \dot{\xi}_{r, j}(t)=-\gamma_{r, j} \xi_{r, j}(t)+\beta_{r, j}\|r(t)\|_{2} \\
& \theta_{r, j}(t)=\xi_{r, j}(t)+\alpha_{r, j}\|r(t)\|_{2},
\end{aligned}
$$

The filter parameters are $\alpha_{r, j} \geq 0$ and $\beta_{r, j} \geq 0$ representing weights for instantaneous and long-term values, respectively, and $\gamma_{r, j}>0$ is the forgetting factor. The evaluation signal $\theta_{r, j}(t)$ is compared to a specific threshold $J_{t h_{r, j}}$ in the decision making process to determine the decision signal $i_{j}$ using the decision logic

$$
\begin{aligned}
& \theta_{r, j}(t)<J_{t h_{r, j}} \Rightarrow i_{j}(t)=0 \Rightarrow \text { no fault } \\
& \theta_{r, j}(t) \geq J_{t h_{r, j}} \Rightarrow i_{j}(t)=1 \Rightarrow \text { fault }
\end{aligned}
$$

The signal $\theta_{r, j}(t)$ is ideally equal to zero or sufficiently small in fault free situations, whereas it shall exceed the threshold $J_{t h_{r, j}}$ when a fault occurs in the system. Hence, the appropriate selection of the values of the free parameters $\alpha_{r, j}, \beta_{r, j}$ or $\gamma_{r, j}$, together with an appropriate threshold $J_{t h_{r, j}}$ essentially influences the performance of the FDD system.

\section{Signal based fault identification}

The decision signals $i_{j}$ can be used to trigger the fault identification process which determines qualitative and quantitative information of the occurred fault. In the case of an actuator jamming, this would be the exact position where the actuator is stuck, while for the runaway fault the runaway rate is an important parameter. In what follows we discuss two identification schemes: (1) for the actuator jamming in null position, and (2) for an actuator runaway with different runaway speeds. In both cases, we consider the important case of elevator actuator faults. 


\section{Actuator jamming at null position}

An elevator jamming in null position appears to be the case which is the most difficult to detect, as the fault can be detected only if a nonzero input signal $u$ acts. Hence, if the fault appears during a cruise or level flight condition with $y=0$ and $u=0$, the residual signal will remain zero and such a fault can not be detected. However, the jamming in null can be detected as soon as any flight maneuver begins, in which case a nonzero residual $r$ result for $u \neq 0$ and $y=0$.

To identify the fault as actuator jamming in null position, a signal based analysis can be performed. This will involve the evaluation signal $\theta_{r, 1}(t)$ for the residual signal $r$ using the Narendra signal evaluation method, as well as similar evaluation signals $\theta_{u}(t)$ and $\theta_{y}(t)$ for the input and output signals, respectively. The corresponding Narendra filters to generate $\theta_{u}(t)$ and $\theta_{y}(t)$ are

$$
\begin{aligned}
\dot{\xi}_{y}(t) & =-\gamma_{y} \xi_{y}(t)+\beta_{y}\|y(t)\|_{2} \\
\theta_{y}(t) & =\xi_{y}(t)+\alpha_{y}\|y(t)\|_{2},
\end{aligned}
$$

and respectively

$$
\begin{aligned}
& \dot{\xi}_{u}(t)=-\gamma_{u} \xi_{u}(t)+\beta_{u}\|u(t)\|_{2} \\
& \theta_{u}(t)=\xi_{u}(t)+\alpha_{u}\|u(t)\|_{2},
\end{aligned}
$$

Here, $\alpha_{y}, \beta_{y}$ and $\gamma_{y}$ and $\alpha_{u}, \beta_{u}$ and $\gamma_{u}$ are the corresponding weights and forgetting factors used in the filters.

An actuator fault is detected if $\theta_{r, 1}(t)$ exceeds the corresponding threshold $J_{t h_{r, 1}}$. In this case, an actuator jamming in null position occurred provided $\theta_{y}(t)$ is sufficiently small, thus satisfies $\theta_{y}(t)<J_{t h_{y}}$ for an appropriate threshold $J_{t h_{y}}$ for zero signals, and simultaneously the signal $\theta_{u}(t)$ exceeds the threshold $J_{t h_{u}}$ for nonzero signals. We can combine the three conditions for $r, y$ and $u$ into a single condition for the identification of an actuator stuck in null position, by defining the classification signal $c_{1}$ as

$$
\begin{aligned}
& c_{1}=1 \quad \text { if } \quad\left(\theta_{r, 1}(t)>J_{t h_{r, 1}}\right) \wedge\left(\theta_{u}(t)>J_{t h_{u}}\right) \wedge\left(\theta_{y}(t)<J_{t h_{y}}\right) \quad \text { is true } \\
& c_{1}=0 \quad \text { if } \quad\left(\theta_{r, 1}(t)>J_{t h_{r, 1}}\right) \wedge\left(\theta_{u}(t)>J_{t h_{u}}\right) \wedge\left(\theta_{y}(t)<J_{t h_{y}}\right) \text { is false }
\end{aligned}
$$

\section{Actuator runaway}

An actuator runaway can usually appear with different velocities depending, for example, on the source of the fault (e.g. hydraulic system, control loop) and the current aerodynamic forces acting on the control surface. However usually it can be assumed that during the occurrence of the fault the runaway velocity remains constant until the actuator reaches is deflection limit. Therefore, the variation rate $\dot{y}$ of the actuator output signal $y$ will be nonzero during the occurrence of the runaway. For monitoring the magnitude of $\dot{y}$, we use, similarly as for the other signals, a Narendra filter of the form

$$
\begin{aligned}
\dot{\xi}_{\dot{y}}(t) & =-\gamma_{\dot{y}} \xi_{\dot{y}}(t)+\beta_{\dot{y}}\|\dot{y}(t)\|_{2} \\
\theta_{\dot{y}}(t) & =\xi_{\dot{y}}(t)+\alpha_{\dot{y}}\|\dot{y}(t)\|_{2},
\end{aligned}
$$

can be used to approximate the norm of $\dot{y}$. For the identification of runaway, $\theta_{\dot{y}}(t)$ is compared to the threshold $J_{t h_{\dot{y}}}$ to decide if the actuator is moving, i.e. $\theta_{\dot{y}}(t)>J_{t h_{\dot{y}}}$ or not, i.e. $\theta_{\dot{y}}(t) \leq J_{t h_{\dot{y}}}$. Note that the latter condition can be used to identify also a jammed actuator in an arbitrary nonzero position. As the condition $\theta_{\dot{y}}(t)>J_{t h_{i j}}$ might also be true for other type of faults (e.g., oscillatory failure), an additional check is required to identify actuator runaways. Assuming a constant velocity $\dot{y}$ in the case of a runaway, we can check that the variance $\sigma_{\dot{y}}$ of the actuator velocity $\dot{y}$ is zero or sufficiently small. This variance is monitored over a time window of length $T_{\sigma_{\dot{y}}}$ and, in the case of runaway, it must be below a threshold $J_{t h, \sigma_{\dot{y}}}$. Note that this condition is also fulfilled during an actuator jamming.

We can combine the three conditions for $r, \dot{y}$ and $\sigma_{y}$ into a single condition for the identification of an actuator runaway, by defining the classification signal $c_{2}$ as

$$
\begin{aligned}
& c_{2}=1 \quad \text { if } \quad\left(\theta_{r, 2}(t)>J_{t h_{r, 2}}\right) \wedge\left(\theta_{\dot{y}}(t)>J_{t h_{\dot{y}}}\right) \wedge\left(\sigma_{\dot{y}}<J_{t h, \sigma_{\dot{y}}}\right) \quad \text { is true } \\
& c_{2}=0 \quad \text { if } \quad\left(\theta_{r, 2}(t)>J_{t h_{r, 2}}\right) \wedge\left(\theta_{\dot{y}}(t)>J_{t h_{\dot{y}}}\right) \wedge\left(\sigma_{\dot{y}}<J_{t h, \sigma_{\dot{y}}}\right) \text { is false }
\end{aligned}
$$

The main challenge in the case of runaway is the need for an early detection and identification of this fault, to prevent significant movements of the corresponding control surface. 


\section{FDD system assessment}

We described in the previous sections a fault detection and identification approach for aircraft actuator faults which is intended to be implemented for a component level actuator fault monitoring. In a real application, several FDD systems as presented in Fig. 4 have to be used for monitoring different actuators/control surfaces. Since the resulting overall FDD system operates jointly with the flight control system, its assessment must be naturally performed employing the augmented nonlinear closed-loop aircraft model. This augmented closed-loop aircraft structure includes the open-loop aircraft, actuators, sensors and a robust stabilizing controller and can be modelled by a nonlinear state-space model description of the form

$$
\begin{aligned}
& \dot{x}_{c l}(t)=F\left(x_{c l}, u_{p}, d, f, \rho_{1}\right) \\
& y_{m}(t)=G\left(x_{c l}, u_{p}, d, f, \rho_{1}\right)
\end{aligned}
$$

where $x_{c l}$ is the state vector of the closed-loop system, $u_{p}$ is the pilot input vector, $y_{m}$ denotes the measured output vector, $d$ is an unknown disturbance vector, $f$ is a vector of actuator fault signals, and $\rho_{1}$ is a constant parameter vector (e.g., mass $m$ and center of gravity $X_{c g}$ ). $F$ and $G$ are suitable nonlinear vector functions. We will not further detail the structure of this system, but we will assume that the closed-loop system is robustly stable over the whole flight domain and for all parameter values.

For the assessment of the performance of the fault monitoring, we consider a single FDD system as in Fig. 4 for an elevator actuator. The residual generator has the LPV gain scheduling form (8), with a realization of $\bar{Q}\left(s, \rho_{2}\right)$ given in $(20)$.

Two identification channels are setup to identify two types of fault signals $f_{j} \in \mathcal{F}_{j}$, for $j=1,2$, where $\mathcal{F}_{1}$ is the class of fault signals leading to actuator jamming in null position, and $\mathcal{F}_{2}$ contains the fault signals leading to actuator runaway. While $\mathcal{F}_{2}$ can be explicitly described to contain maximum amplitude step signals with initial slopes ranging in a given interval of values, $\mathcal{F}_{1}$ is only implicitly defined by the condition that the actuator output is stuck in null position.

Typical performance requirements used in the industry are expressed in terms of the false alarm rate (FAR), the missed detection rate (MDR) or the detection time performance (DTP). Due to the presence of unknown external signals (e.g., pilot inputs, wind disturbances) and parametric uncertainties these performance criteria are hardly computationally tractable, because their computations rely on expensive simulation runs and involve the solution of worst-case optimization problems with semi-infinite constraints. To overcome this computational bottleneck, we propose in what follows an assessment approach which, when applicable, provides suitable thresholds for decision making which completely avoid false alarms and missed detections.

\section{A. Robustness assessment}

To define computable approximations of the FAR and MDR criteria, simplifying assumptions are necessary. Instead of arbitrary signals $u_{p}$ and $d$, in practical applications it is sufficient to use bounded input signals in a given finite class $\mathcal{U}$ and bounded disturbance signals in a finite class $\mathcal{D}$. A false alarm bound for the fault $f_{j}$ can be defined as

$$
\begin{aligned}
& J_{t h, j}^{f}=\sup _{\rho \in \Pi} \max _{t \leq t_{f i n}} \theta_{r, j}(t) \\
& u_{p} \in \mathcal{U} \\
& d \in \mathcal{D} \\
& f=0
\end{aligned}
$$

Here, $t_{\text {fin }}$ is chosen in accordance with the duration of maneuvers. To avoid false alarms, the threshold $J_{t h_{r, j}}$ used in the decision blocks must be chosen such that $J_{t h_{r, j}}>J_{t h, j}^{f}$.

The minimization of MDR addresses the fault detectability condition (ii) of the RFDP and can be seen equivalent to maximize the so-called detection bound defined as

$$
\begin{gathered}
J_{t h, j}^{d}=\inf _{\rho \in \Pi} \max _{t \leq t_{\text {detec }}} \theta_{r, j}(t) \\
u_{p} \in \mathcal{U}_{j} \\
d=0 \\
f \in \mathcal{F}_{j}
\end{gathered}
$$


Here, $\mathcal{U}_{j}$ is the class of inputs used for a specific maneuver during which the fault $f_{j} \in \mathcal{F}_{j}$ occurs and has to be detected within a specified detection time $t_{\text {detec }}$. Note that the detection times for different fault signals in $\mathcal{F}_{j}$ may be different.

As a robustness measure of the fault detection performance, the smallest detection gap $J_{t h, j}^{d}-J_{t h, j}^{f}$ can be used, or alternatively the detection factor defined as

$$
\nu_{j}:=\frac{J_{t h, j}^{f}}{J_{t h, j}^{d}}
$$

If $J_{t h, j}^{d}-J_{t h, j}^{f}>0$ (or equivalently $\nu_{j}<1$ ), a constant threshold can be chosen to serve for fault detection, ensuring no false alarms and no missed detections.

For the computation of $J_{t h, j}^{f}$ and $J_{t h, j}^{d}$, solving global worst-case optimization problems to find the worst-case parameter combinations appears to be the most adequate choice. However, less demanding computational approaches can be used, as a gridding based worst-case search over the flight envelope and parameter space, or Monte-Carlo simulations, to determine approximations of the upper bound $J_{t h, j}^{f}$ and lower bound $J_{t h, j}^{d}$.

The detection time of a fault $f_{j}$ can be determined as

$$
t_{d, j}=\min _{t \leq t_{f i n}}\left\{t \mid \theta_{r, j}(t)>J_{t h_{r, j}}\right\} .
$$

Since the required detection time $t_{d e t e c, j}$ may depend on the characteristics of the fault $f_{j}$ (e.g., magnitude or rate), it is reasonable to use the detection time factor $\vartheta_{j}$ instead of the absolute detection time for a worst case search:

$$
\vartheta_{j}=\sup _{\substack{\rho \in \Pi \\ u \in \mathcal{U}_{j} \\ d \in \mathcal{D} \\ f_{j} \in \mathcal{F}_{j}}} \frac{t_{d, j}}{t_{\text {detec }, j}}
$$

\section{B. Decision thresholds}

\section{Threshold for residual signal}

To avoid both false alarms and missed detections, the threshold $J_{t h, j}$ used in the decision block must be chosen such that

$$
J_{t h, j}^{f} \leq J_{t h_{r, j}}<J_{t h, j}^{d}
$$

Such a choice is always possible by increasing the size of minimum amplitude of faults to be detected. A choice of threshold as $J_{t h_{r, j}}=J_{t h_{r, j}}^{f}+\varepsilon_{r, j}$, with a small positive gap $\varepsilon_{r, j}$, allows the reliable detection of smallest detectable faults.

\section{Thresholds for detecting actuator jamming}

In the case of the actuator jamming in null position, the actuator output $y$ is zero for all values of the actuator input $u$. The detection of such a fault is only possible if simultaneously the actuator input signal is significantly different of zero. Since the output evaluation signal $\theta_{y}(t)=0$ (or nearly zero), the choice of a small threshold $J_{t h_{y}}>0$ to check that $\theta_{y}(t) \leq J_{t h_{y}}$ is straightforward. However, to simultaneously check that $u \neq 0$ via $\theta_{u}(t) \geq J_{t h_{u}}$, the choice of an appropriate threshold $J_{t h_{u}}$ is necessary, which takes into consideration the least norm of the control signals during a turn maneuver in the absence of fault, over all flight conditions and all parameter values. Therefore $J_{t h_{u}}$ has to fulfill the conditions

$$
\begin{aligned}
& 0<J_{t h, u} \leq \inf _{\rho \in \Pi} \max _{t<t_{f i n}} \theta_{u}(t) \\
& u_{p} \in \mathcal{U}_{1} \\
& d \in \mathcal{D} \\
& f=0
\end{aligned}
$$

where $\mathcal{U}_{1}$ is the class of pilot inputs used to perform the turn maneuver. Thus, the determination of $J_{t h_{u}}$ involves the solution of a worst-case global minimization problem to determine the upper bound in the right-hand side of (38). 


\section{Thresholds for detecting actuator runaway}

In the case of an actuator runaway, the early detection and identification of the fault is required, before the variation of the control surface deflection is bellow a certain acceptable value. Thus, the identification of the runaway must occur during the output signal amplitude rise time and requires the checking of the conditions $\dot{y} \neq 0$ and $\dot{y}=$ constant. The latter condition tacitly assumes that the runaway occurs with a constant rate of variation $\dot{y}$ during the whole detection time. To check the condition $\dot{y} \neq 0$, a threshold $J_{t h_{\dot{y}}}$ is used to verify that $\theta_{\dot{y}}(t) \geq J_{t h_{\dot{y}}}$. The value of the threshold $J_{t h_{\dot{y}}}$ must fulfill the condition

$$
\begin{gathered}
0 \leq J_{t h, \dot{y}}<\inf _{\rho \in \Pi} \max _{t \leq t_{f i n}} \theta_{\dot{y}}(t) \\
u_{p}=0 \\
d \in \mathcal{D} \\
\quad f \in \mathcal{F}_{2}
\end{gathered}
$$

which ensures that the least effect of the runaway on $\theta_{\dot{y}}$ is taken into account and also $\dot{y} \neq 0$ ( $\dot{y}=0$ would indicate an actuator jamming). To check $\dot{y}=$ constant, the variance $\sigma_{\dot{y}}$ of the actuator's velocity $\dot{y}$ is monitored over a time window of length $T_{\sigma_{\dot{y}}}$. The variation rate is constant, if $\sigma_{\dot{y}} \leq J_{t h, \sigma_{\dot{y}}}$, where the threshold $J_{t h, \sigma_{\dot{y}}}$ has to fulfill the condition

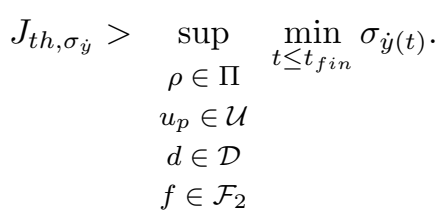

to account for the disturbances $\mathcal{D}$ and inputs $\mathcal{U}$ influencing $\sigma_{\dot{y}}$.

\section{Application examples}

In this section we describe the application of the proposed approach to a nonlinear model of a closedloop aircraft including a nonlinear control law ensuring robust stability over the whole flight envelope. The aircraft model has been previously used in a flight control law clearance study and is described in ${ }^{4}$. The bounds of the flight envelope (altitude, speed) and of the mass and center of gravity position are given in Table 2 .

\begin{tabular}{|l|c|c|c|}
\hline Parameter & Variable & Minimum & Maximum \\
\hline Altitude (ft) & $h$ & 0 & 41000 \\
Calibrated airspeed (kts) & $V_{c a s}$ & 120 & 325 \\
Mass (t) & $m$ & 120 & 233 \\
Center of gravity (\%) & $X_{c g}$ & 17 & 41 \\
\hline
\end{tabular}

Table 2. Values of the envelope bounds

While the limits of the altitude $h$ and the mass $m$ in Table 2 are fixed, the limits of the center of gravity $X_{c g}$ depend on the actual mass, while the limits of the calibrated airspeed $V_{c a s}$ depend on the actual mass and altitude. This leads to somewhat lower bounds for these parameters depending on the concrete flight condition.

The nonlinear closed-loop aircraft model has been augmented with an FDD system as in Fig. 4, which includes a LPV residual generator determined using the LPV actuator model presented in section II. Further components of the FDD system are the blocks for residual evaluation, decision making and fault identification of two actuator faults: jamming at null position and runaway.

The detection of actuator faults is an important issue for aircraft certification. Typical actuator faults to be detected are jamming with the actuator stuck at a constant value, actuator runaway with different rates of variations, or oscillations around the measured values. In this study the actuator stuck at null position and the actuator runaway with different runaway rates are investigated. The exact fault specifications including the required detections times are given in Table 3. 


\begin{tabular}{|l|l|l|}
\hline Failure type & Specification & Detection time requirement \\
\hline Jamming: $\left(\mathcal{F}_{1}\right)$ & at null position during a turn maneuver & before turn maneuver is over \\
Runaway: $\left(\mathcal{F}_{2}\right)$ & with up to $\pm 50 \mathrm{deg} /$ sec during level flight & before elevator deflection exceeds $\pm 2.5 \mathrm{deg}$ \\
\hline
\end{tabular}

Table 3. Definition of fault sets $\mathcal{F}_{j}$ for the actuator faults to be detected

\section{A. Setup of an enhanced LPV detector}

The implemented residual generator (22) provides a residual signal to serve for decision making on the presence and absence of actuator faults. To allow a fast detection of the occurring faults, the dynamics of the detector is specified with $a=10$. The nominal gain is set to a mean value of $k_{0}=14$. As the actual nonlinear actuator includes physical rate and position limitations which are activated during certain aircraft maneuvers, it is important to consider these limitations also in the setup of the residual generation filter. Upper and lower position limitations $u_{\max }$ and $u_{\min }$ on the actuator input $u(t)$ and a rate limitation $\dot{u}_{\max }$ on the magnitude of $\dot{u}(t)$ are included in the residual filter, so that instead of $u$, the variable

$$
\widetilde{u}=R\left(u, u_{\max }, u_{\min }, \dot{u}_{\max }\right)
$$

is the actual input applied via a nonlinear mapping $R$. The specific values of the limits are taken from the nonlinear actuator model, where $\dot{u}_{\max }=30 \mathrm{deg} / \mathrm{s}, u_{\max }=15 \mathrm{deg}$ and $u_{\min }=-30 \mathrm{deg}$.

\section{B. Setup of parameters of the FDD system}

In the following, we discuss the setup of the parameters of the FDD system for the identification of two types of elevator faults.

\section{Elevator actuator jamming in null position}

Common civil aircraft configurations employ two independent elevators, ensuring the aircraft's maneuverability even when the actuator of one elevator is jammed. The negative effects of jamming are well-known. The increased drag leads to increased fuel consumption. A challenging case is an actuator jamming at small deflections (e.g., in null position) as it may remain undetected for a long period of time. For example, during a cruise phase, this fault is practically undetectable, due to the small inputs $u$ used. Therefore, the detection of actuator jamming in null position is addressed in what follows during a turn maneuver, where both the altitude and the heading of the aircraft are changing.

To select an appropriate threshold $J_{t h_{r, 1}}$, the false alarm bound $J_{t h, 1}^{f}$ and the detectability bound $J_{t h, 1}^{d}$ have to be determined for a specific choice of the parameters of the evaluation filter (see Table 4). $J_{t h, 1}^{f}$ is determined for level flight and turn situations in the flight envelope, and additionally, for a finite set of typical aircraft maneuvers $\mathcal{U}$ and typical disturbances $\mathcal{D}$. The maneuvers $\mathcal{U}$ include, for example, piloted flights with various pilot inputs (longitudinal/lateral stick doublets, pedal inputs, nose up/nose down demands) or typical navigation maneuvers (level flight, flight path angle target mode, yaw angle target mode, speed change, steady sideslip, coordinated turn, etc.). Typical disturbance inputs used to define $\mathcal{D}$ are random wind speeds with given variances. The detectability bound $J_{t h, 1}^{d}$ for the defined jamming is determined using a worst-case global optimization relying on closed-loop simulations within the whole flight envelope. While for the false alarm bound a value of $J_{t h, 1}^{f}=3.27$ was determined, the lowest value of the detectability bound was determined at $J_{t h, 1}^{d}=3.42$, leading to a detection factor of $\nu_{1}=0.96$. Therefore a constant threshold $J_{t h_{r, 1}}$ can be selected, which allows a reliable detection of the jamming in null position in the whole flight envelope without triggering false alarms. The threshold for the FDD system was chosen as $J_{t h_{r, 1}}=3.37$, which includes an additional gap of $\epsilon_{r, 2}=0.1$ added to the false alarm bound. The settings of the FDD system parameters for the detection and identification of an actuator jamming are given in Table 4.

The instantaneous weight to determine the evaluation signals $\theta_{r, 1}$ is set to 0 so that the signal is not occasionally increasing due to disturbances with greater magnitudes. The forgetting factor $\gamma_{r, 1}$ has to be set to a small value to ensure that the evaluation signal $\theta_{r, 1}$ exceeds the threshold in the required detection time. The parameter setting for the fault identification is chosen in a way that a fast crossing of the defined threshold is guaranteed so that the jamming of the actuator is detected before the turn maneuver is over 


\begin{tabular}{|c|c|c|c|c|c|c|c|}
\hline $\begin{array}{c}\text { Short term } \\
\text { weights }\end{array}$ & Value & $\begin{array}{c}\text { Long term } \\
\text { weights }\end{array}$ & Value & $\begin{array}{c}\text { Forgetting } \\
\text { factors }\end{array}$ & Value & Thresholds & Value \\
\hline$\alpha_{r, 1}$ & 0 & $\beta_{r, 1}$ & 1 & $\gamma_{r, 1}$ & 0.001 & $J_{t h_{r, 1}}$ & 3.37 \\
$\alpha_{u}$ & 0 & $\beta_{u}$ & 1 & $\gamma_{u}$ & 0.01 & $J_{t h_{u}}$ & 1 \\
$\alpha_{y}$ & 0 & $\beta_{y}$ & 1 & $\gamma_{y}$ & 0.9 & $J_{t h_{y}}$ & 0.01 \\
\hline
\end{tabular}

Table 4. FDD parameter setting for actuator jamming detection in null position

(to comply with the defined detection time requirements). The detection time factor $\vartheta_{1}$ was determined by worst-case optimization with a value of $\vartheta_{1}=0.96$, indicating that the jamming can be detected in the whole envelope in the required time.

\section{Elevator actuator runaway}

At signal level, the actuator runaway can be described as an output signal driven directly by the fault, i.e., $y(t)=f(t)$, where the rate of variation $\dot{f}(t)$ of the fault $f(t)$ is constant and nonzero. We consider the case when the magnitude $|\dot{f}(t)|$ can take values in an interval $[1,50] \mathrm{deg} / \mathrm{s}$, ranging from slow to very fast rates. The runaway results in an undesired pitch maneuver and may significantly degrade the aircraft controllability. In all cases, it will increase drag and thus the fuel consumption, and adds unnecessary workload to the pilot. Hence, a fast detection of runaway is desirable. Typically, the detection of the runaway must be accomplished before the elevator position exceeds $\pm 2.5 \mathrm{deg}$ from its trimmed value. This aim results in a required detection time $t_{\text {detec }}$ depending on the actual fault rate $\dot{f}$. For setting the decision threshold, the values of the false alarm bound $J_{t h, 2}^{f}$ and detection bound $J_{t h, 2}^{d}$ have been determined. For the specific choice of the parameters of the evaluation filter (see Table 5 ), these values are $J_{t h, 2}^{f}=0.48$ and $J_{t h, 2}^{d}=2.38$, which lead to a very satisfactory detection factor of $\nu_{2}=0.19$. This low value is mainly due to the highly dominant contribution of the fault signal during the occurrence of the runaway, while the influences of the disturbances $d \in \mathcal{D}$ and the inputs $u \in \mathcal{U}$ during a level flight in the envelope and the dedicated maneuvers are rather low. Based on this result the threshold was set to $J_{t h_{r, 2}}=0.51$, which includes an additional gap of $\epsilon_{r, 2}=0.03$ added to the false alarm bound $J_{t h, 2}^{f}$. The detailed values of the FDD system parameters for the runaway detection are listed in Table 5, where the parameter setting for the fault identification is chosen in a way that a fast crossing of the defined thresholds is guaranteed so that the fault detection is finished within the required detection time.

\begin{tabular}{|c|c|c|c|c|c|c|c|}
\hline $\begin{array}{c}\text { Short term } \\
\text { weights }\end{array}$ & Value & $\begin{array}{c}\text { Long term } \\
\text { weights }\end{array}$ & Value & $\begin{array}{c}\text { Forgetting factors/ } \\
\text { Observation window }\end{array}$ & Value & Thresholds & Value \\
\hline$\alpha_{r, 2}$ & 1 & $\beta_{r, 2}$ & 0 & $\gamma_{r, 2}$ & 0 & $J_{t h_{r, 2}}$ & 0.51 \\
$\alpha_{\dot{y}}$ & 0 & $\beta_{\dot{y}}$ & 1 & $\gamma_{\dot{y}}$ & 0.2 & $J_{t h_{\dot{y}}}$ & 1 \\
$T_{\sigma_{\dot{y}}}$ & 0.03 & $J_{t h_{\sigma_{\dot{y}}}}$ & 0.01 \\
\hline
\end{tabular}

Table 5. FDD parameter setting for actuator runaway detection

The detection time analysis and the determination of the detection time factor results in a value of $\vartheta_{2}=0.8$, so that even when the runaway appears at high velocities (i.e., near to $\dot{f}= \pm 50 \mathrm{deg} / \mathrm{s}$ ) the detection is finished within the required time. For example, for $\dot{f}= \pm 50 \mathrm{deg} / \mathrm{s}$, this means that the absolute detection time must be below $50 \mathrm{~ms}$, as at this time the elevator exceeds the deflection limit of \pm 2.5 deg given by the detection time criteria.

\section{Evaluation of worst-case search techniques}

To demonstrate the effectiveness and reliability of the applied optimization-based worst-case search to determine suitable values for the detection thresholds, we performed a comparison of this approach with the Monte-Carlo simulation and gridding based search methods. We discuss here only the determination of the 
detectability bound $J_{t h, 1}^{d}$ and the corresponding worst-case of the detection time factor $\vartheta_{1}$ for the actuator jamming in null position. However, qualitatively similar results are to be expected for the false alarm bound $J_{t h, 1}^{f}$ as well. The optimization-based worst-case search has been performed using the optimization environment MOPS (Multi-Objective Parameter Search) of DLR. ${ }^{2}$ Specifically, the differential evolution global search method has been employed, which allows to perform many function evaluations in parallel. Thus, parallel computations have been employed to alleviate the associated computational burden due to expensive simulation runs. Table 6 shows the results for the computation of $J_{t h, 1}^{d}$ and $\vartheta_{1}$ and the corresponding required numbers of function evaluations (NFE). The optimization runs were performed on a Linux-cluster consisting using 16 CPUs. An optimization run involving about 1000 function evaluations takes around 30 minutes.

\begin{tabular}{|l|cc|cc|}
\hline Method & $J_{t h, 1}^{d}$ & NFE & $\vartheta_{1}$ & NFE \\
\hline Optimization-based search & 3.43 & 1400 & 0.94 & 1000 \\
Monte Carlo simulation & 3.83 & 1400 & 0.70 & 1000 \\
Gridding-based search & 3.77 & $\approx 1400$ & 0.74 & $\approx 1000$ \\
\hline
\end{tabular}

Table 6. Efficiency of different methods for detection bound and detection time determination

As it can be observed, the use of the Monte Carlo simulation with the same number of function evaluations as performed with the optimization-based worst-case search (i.e., 1400), leads to a significantly larger value of the estimated $J_{t h, 1}^{d}$. In some cases, this could lead to missed detections when employing this larger value of the bound for the determination of the decision threshold. Using a uniform grid in the 4-dimensional parameter space corresponding to about 1400 points, leads to a comparable high value of the detection bound. For this example, about $10^{4}$ function evaluations were necessary for the Monte Carlo method to determine a worst-case which nearly fits the value determined via optimization. However, this represents an almost seven times larger computational effort. Interestingly, the grid-based search with $10^{4}$ uniformly generated grid points misses to find a better value than with 1400 grid points, which illustrates the potential danger of discrete search based approaches in missing worst-case points lying between the grid points. It appears, that the optimization-based worst-case search relying on global optimization techniques is the only generally applicable method to reliably determine the false alarm and detection bounds.

\section{Using a reduced set of scheduling variables}

When the components of $\rho_{1}=\left(m, X_{c g}\right)$ are not measurable, we have to employ $\widetilde{k}\left(\rho_{2}\right)$ instead $k(\rho)$. It appears that for fault detection purposes, it is possible to determine a robust approximation $\widetilde{k}\left(\rho_{2}\right)$ of $k(\rho)$ in a simple way by solving

$$
\left\{m^{*}, X_{c g}^{*}\right\}=\arg \min _{m, X_{c g}} J_{t h_{r, 1}}^{f}
$$

and setting $\widetilde{k}\left(\rho_{2}\right)=k\left(\rho_{1}^{*}, \rho_{2}\right)$, where $\rho_{1}^{*}=\left(m^{*}, X_{c g}^{*}\right)$. The replacement of $k(\rho)$ by $\widetilde{k}\left(\rho_{2}\right)$ in the expression of the fault detection filter $(22)$ has practically no effects on the detection times. For example, for the detection of the elevator actuator jamming in null position, the worst-case detection time becomes 48.85 sec when using $\widetilde{k}\left(\rho_{2}\right)$, instead of $48.8 \mathrm{sec}$ when using $k(\rho)$.

\section{Conclusion}

In this paper we proposed a model-based methodology for the design of FDD systems for monitoring aircraft actuator faults. The main elements of the proposed methodology are: (1) the development of suitable LPV models of the underlying actuator, (2) the synthesis of LPV residual generators for robust fault detection, (3) the setting up of the fault evaluation and decision making blocks, (4) the determination of detection thresholds, and (5) the robustness assessment. While the synthesis of the LPV residual generator relies on a fault monitoring approach at component (actuator) level, the tuning of the overall FDD system parameters and the final robustness analysis involve the closed-loop aircraft. The assessment of the robustness of the FDD system is supported by various optimization-based worst-case computations, as for example, the determination of false alarm or detection bounds to serve for choosing suitable detection thresholds. The 
high computational burden involved by the parameter tuning via multi-objective optimization, and especially, by the robustness assessment via global worst-case search, can be alleviated by extensively using parallel computational techniques.

The proposed methodology has been applied for the design of an FDD system aiming the identification of two types of elevator actuator faults: jamming in null position and runaway. This FDD system employs a common residual generator designed using recently developed LPV synthesis techniques, and separate signal based fault identification channels, one for each type of considered faults. The proposed FDD system structure is generic, being able to cope with other types of fault, as jamming in an arbitrary position or various oscillatory failures. The resulting FDD system has been set up to guarantee zero values of FAR and MDR criteria. Also the requirements regarding the bounds on the fault detection times are completely fulfilled in presence of parametric uncertainties over the whole range of values. Additional performance improvements can be expected by performing an integrated tuning of all free parameters of the FDD system, using an multi-objective optimization based approach. This aspect will be addressed in a future work.

\section{Acknowledgment}

This work was performed in the framework of the European ADDSAFE Project: Grant agreement no.: FP7-233815.

\section{References}

${ }^{1}$ E. A. Garcia, A. Zolghadri, P. Goupil, L. Lavigne, and P. Simon. Nonlinear observer-based OFC detection for A380 aircraft. In Proc. of 7th IFAC Symposium on Fault Detection, Supervision and Safety of Technical Processes, Barcelona, Spain, 2009.

${ }^{2}$ H.-D. Joos, J. Bals, G. Looye, K. Schnepper, and A. Varga. A multi-objective optimisation-based software environment for control systems design. Proc. of CACSD'2002, Glasgow, UK, 2002.

${ }^{3}$ H. Pfifer and S. Hecker. Generation of optimal linear parametric models for LFT-based robust stability analysis and control design. In Proceedings of IEEE Conference on Decision and Control, Cancun, Mexico, 2008.

${ }^{4}$ G. Puyou and Y. Losser. Clearance benchmark for a civil aircraft. In A. Varga, A. Hansson and G.. Puyou (Eds.) Optimization Based Clearance of Flight Control Laws, Lecture Notes in Control and Information Science, vol. 416, SpringerVerlag, Berlin, (to appear 2011).

${ }^{5}$ A. Varga. On parametric solution of fault detection problems. In IFAC 2011 World Congress, Milano, Italy (submitted), 2011.

${ }^{6}$ K. Zhou, J. C. Doyle, and K. Glover. Robust and Optimal Control. Prentice Hall, 1996.

${ }^{7}$ G. Vinnicombe. Frequency domain uncertainty and the graph topology. IEEE Trans. Automat. Control, 38:1371-1383, 1993. 